\title{
Infrared thermography measurements on ablative thermal protection systems for interplanetary space vehicles
}

\author{
by C. O. Asma*, B. Helber*, L. Di Lello*, F. Panerai and T. Magin*
}

*Aeronautics \& Aerospace Dept., von Karman Institute for Fluid Dynamics, Rhode-St-Genese (Brussels), 1640 Belgium, asma@vki.ac.be

\begin{abstract}
Ablative thermal protection material design needs suitable ground testing under high enthalpy flow conditions using intrusive and non-intrusive measurement techniques. The main objective is to develop the ground testing environment adapted for the study of ablative materials at high heat flux levels and provide a database on precise information about the materials to be characterised. This paper presents the studies aimed at the testing of cork based ablative material samples in the Plasmatron Facility of the von Karman Institute, up to cold wall heat flux levels of $3 \mathrm{MW} / \mathrm{m}^{2}$. Infrared thermography has been extensively used in addition to other techniques for the characterization of ablative materials.
\end{abstract}

\section{Introduction}

When a space vehicle is to make an entry into the atmosphere of the destination planet, it has to be protected from severe aerothermodynamic heating. The extremely high amount of kinetic and potential energy that the space vehicle has at the beginning of its atmospheric flight are transformed into thermal energy while the space vehicle lands down. Thermal protection system (TPS) materials are used to shield hypersonic aerospace vehicles from the severe aerodynamic heating encountered during atmospheric entry. Proper design of TPS is one of the most critical stages of the design of the space vehicles as it is indeed a complicated optimisation problem between many scientific restrictions. On one hand, the TPS has to be light enough, but on the other hand it has to guarantee the protection of the vehicle and its payloads from extreme temperatures. The aerodynamic constraints on the external shape of the space vehicle bring additional difficulties on designing the TPS [1].

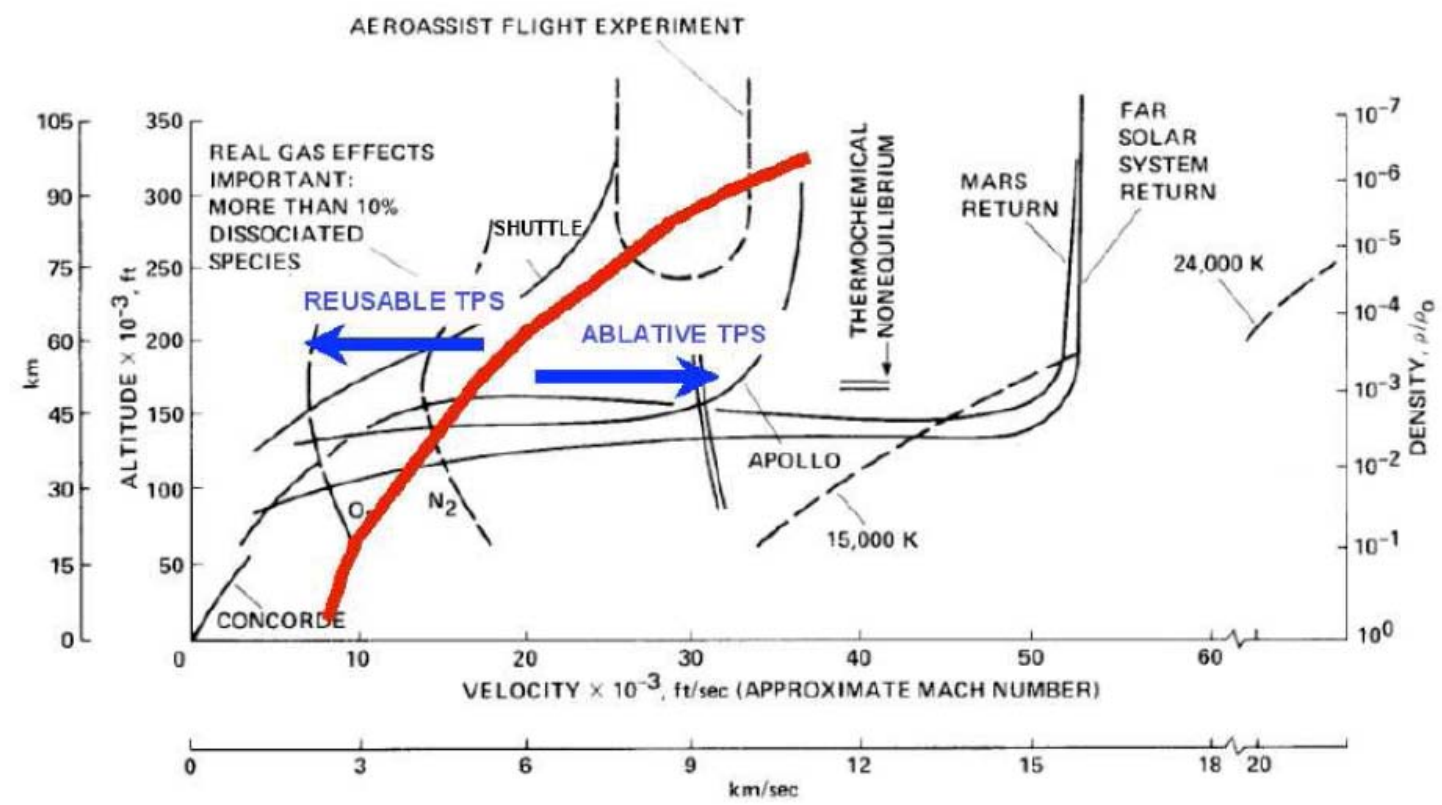

Fig. 1. Atmospheric entry environment, after [2]

The materials used for thermal protection can be reusable or ablative. Reusable TPS materials experience no or minor change in mass and physical properties and are generally preferred at relatively mild re-entry environments whereas, 
on the contrary, ablative TPS materials are used in extremely high temperature environments and experience phase change and mass loss during re-entry. Ablative materials are preferred for inter-planetary missions, or in general for missions with entry velocity higher than $7 \mathrm{~km} / \mathrm{s}$ (see Fig. 1). Proper characterization of ablative thermal protection materials is of utmost importance for an optimum design of space vehicles. The von Karman Institute has started a basic research program to develop the capability to test and characterize ablative materials at the VKI-Plasmatron facility. In this study, different ablative material samples based on graphite and cork are tested to perform the measurements.

\section{Physics of Ablation}

Ablative material is designed to slowly burn away in a predicted manner, so that heat energy can be kept away from spacecraft by the ablative process gases, while the remaining solid material insulates the vehicle from superheated gases. The charring ablators constitute one of the most important classes of TPS, because they provide efficient protection system through a variety of heat-absorbing mechanisms: initially, the material absorbs the heat it is exposed to and its temperature rises. As it continues to increase, the material undergoes thermal degradation, so that pyrolysis gases are formed and a porous carbonaceous char remains. The gases are heated as they percolate toward the surface thus transferring some energy from the solid to the gas; furthermore, the injection of the gases into the boundary layer alters its properties (undergoing chemical reactions with it), typically resulting in a reduction in convective heating, and the char layer acts as an insulator, and as it becomes thicker, the heat flux to the degraded material is reduced. Chemical reactions between the surface material and boundary layer species result in a consumption of the char leading to surface recession; these reactions can be exothermic (oxidation, at relatively low temperature) or endothermic (sublimation, at higher temperature) and have an important impact on the net energy to the surface. A schematic representation of the whole ablation process is given in Fig 2.

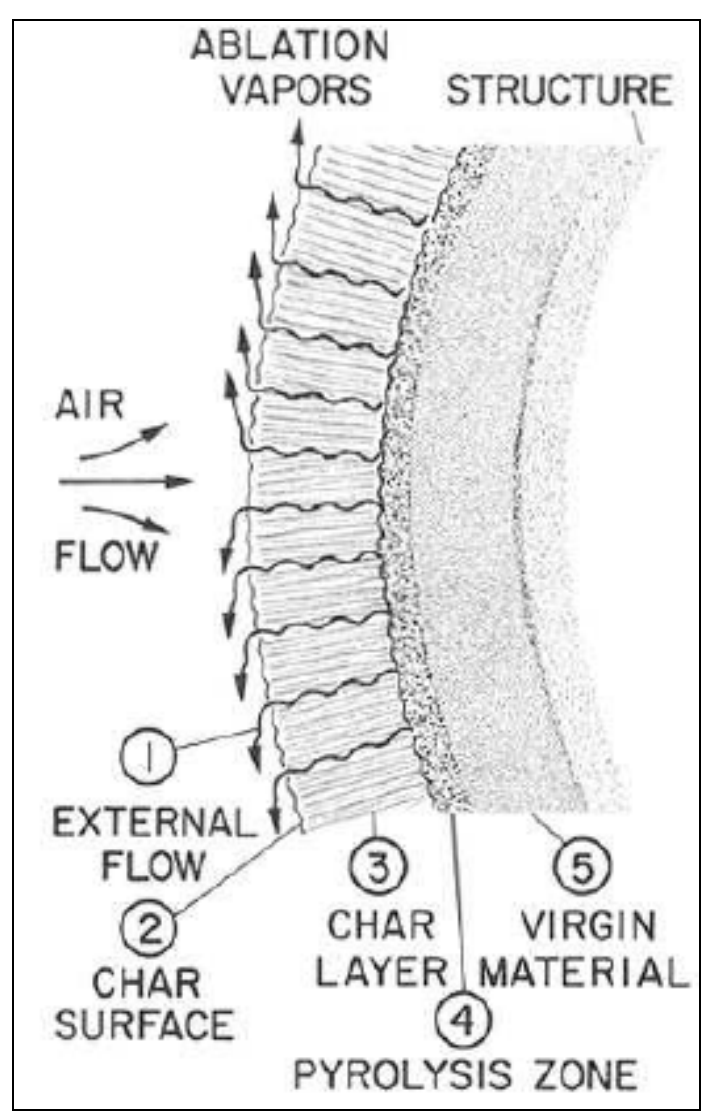

Fig. 2. Charring ablation process, after [3]

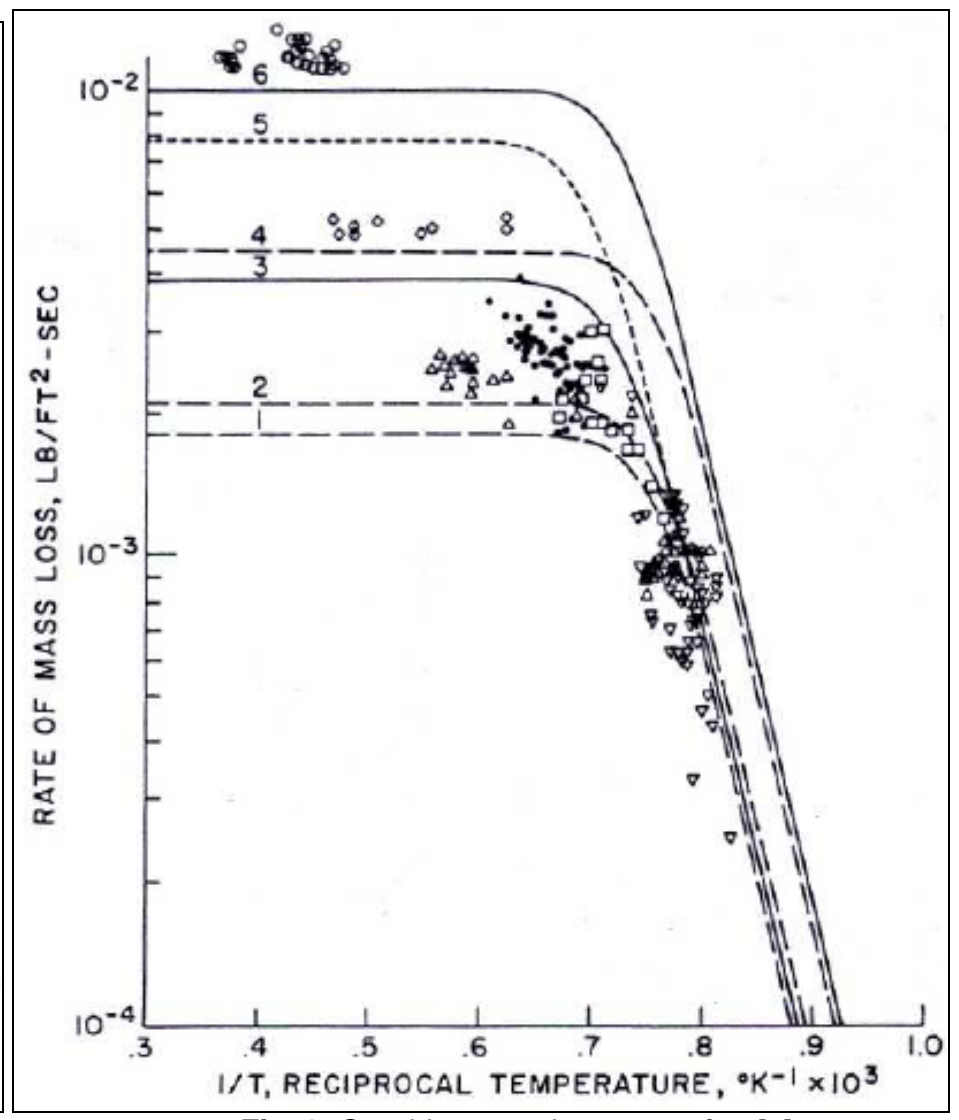

Fig. 3. Graphite mass loss rate, after [4]

The ablative material to be used in space vehicles should be carefully selected such that it must have high emissivity at high temperatures, high specific heat, low surface catalycity, low density and low thermal conductivity. The advantage of high temperature ablators is that they retain their shape in extreme environments; a low density material assures low TPS mass fraction, and it should be apparent that TPS mass fraction does not depend only on the peak heat 
flux, but is also a strong function of the total integrated heat load during the entry (Fig 1.3) [4]. Moreover, in order to choose a suitable ablative material, one should consider a material that ablates very slowly and in a controlled manner. In all cases, selecting a set of ablative material is a balancing act between vehicle shape and size, maximum temperature, heat load requirements and entry speed.

\section{Experimental Setup}

The VKI Plasmatron facility is an inductively-coupled plasma (ICP) wind tunnel equipped with $1.2 \mathrm{MW}, 400 \mathrm{kHz}$, high frequency generator and a torch of $160 \mathrm{~mm}$ diameter [5]. In order to achieve higher heat flux, a converging nozzle can be attached to the torch of the Plasmatron. With the use of this converging nozzle the flow can be concentrated on the stagnation point of the heat flux probe without burning the probe holder and hence higher heat flux values can be achieved. The VKI Plasmatron facility is the most powerful inductively coupled plasma generator in the world and offers a unique opportunity to test ablative materials associated with a collection of measurement techniques. An ICP is a type of plasma source in which the energy is supplied by electrical currents which are produced by electromagnetic induction that is by time varying magnetic fields. It creates high purity plasma flow with heat fluxes up to $10 \mathrm{MW} / \mathrm{m}^{2}$. Since there is no contact between plasma formation and electrodes (compared to typical arc-jet type wind tunnels), the plasma jet is free of contaminations coming from electrodes spluttering. The results is an ideal environment to reproduce the chemistry developing around the TPS during the re-entry. The Plasmatron facility can produce plasmas of argon, air, $\mathrm{N}_{2}$ and $\mathrm{CO}_{2}$, however, air is preferred for this study.

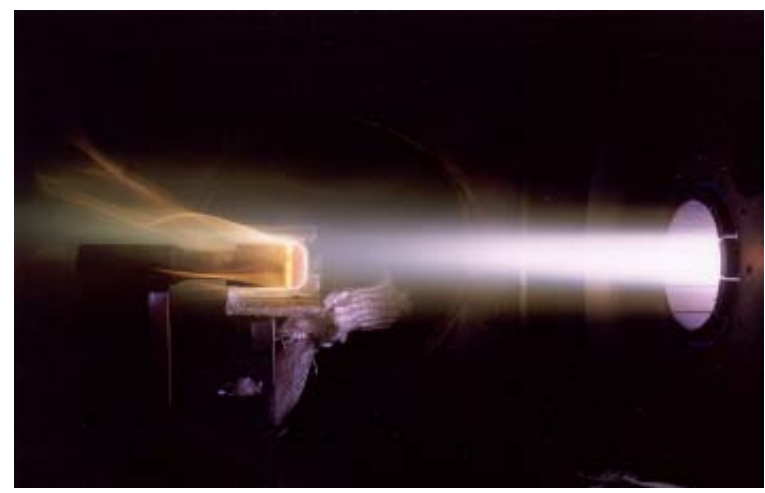

Fig. 4. Test of ablative material in the VKI Plasmatron facility

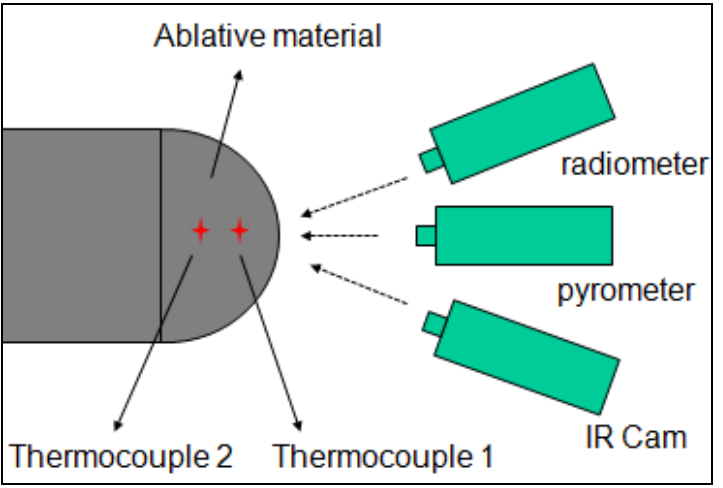

Fig. 5. Front surface temperature measurement

The experimental set up mainly consists of a two-colour pyrometer, an infrared camera, a radiometer, a heat flux probe, thermocouples and a data acquisition system. Before starting the measurements, the heat flux probe is installed, the rotameter is calibrated and the plasma operating conditions are determined: working gas, gas mass flow rate, static pressure and power of the plasma generator. High speed camera visualisations, digital imaging and emission spectroscopy are the other techniques that are applied during the test campaign. A summary of all the measurement techniques applied can be seen in Fig. 6. In this article, the attention is focused on the front surface temperature measurements by infrared thermography.

The two-color pyrometer (Raytek Marathon Series MR1SC with a temperature range of $1000-3000^{\circ} \mathrm{C}$ ) is used to measure the surface temperature of the sample during the test. A two-color pyrometer measures the IR radiation over two narrow overlapping wavebands around 1 micron. If the emissivity, $\varepsilon$, of the material is considered constant over the two wavelengths, an absolute temperature measurement can be determined from a Planck's law analysis for a gray body. The acquisition speed is $1 \mathrm{~Hz}$. The pyrometer is calibrated using a blackbody radiation source, to take into account the effect of the window. The uncertainty is $\pm 10^{\circ} \mathrm{C}$.

A FLIR Thermacam SC3000 infrared camera is used during all experimental activities. This is a self-cooling infrared camera that measures and images the emitted infrared radiation from an object. It is used together with the ThermaCAM Researcher 2001 software (installed on a Microsoft Windows based CPU). The camera works in the range of $8-9 \mu \mathrm{m}$ wavelength. The infrared camera can measure temperatures varying between -20 and $1500^{\circ} \mathrm{C}$ (assuming an emissivity of 1.0), however the accuracy varies as a function of the temperature range selected. The optical access to the test section is a ZnSe window. The camera is not calibrated for the temperature range expected for Plasmatron conditions.

The infrared radiometer Heitronics KT 19.XX is used as an additional front surface temperature information source and it is located in front of a KRS-5 window. The radiometer functions in the wavelength range of 0.6 to $40 \mu \mathrm{m}$, and provides as output the integrated thermal radiation over this spectrum within a temperature range of 0 to $3000^{\circ} \mathrm{C}$. The radiometer is calibrated using a black body radiation source to take the transmissivity of the KRS-5 window into account. The acquisition rate is $1 \mathrm{~Hz}$. The raw radiometer data assumes an emissivity of 1 . 


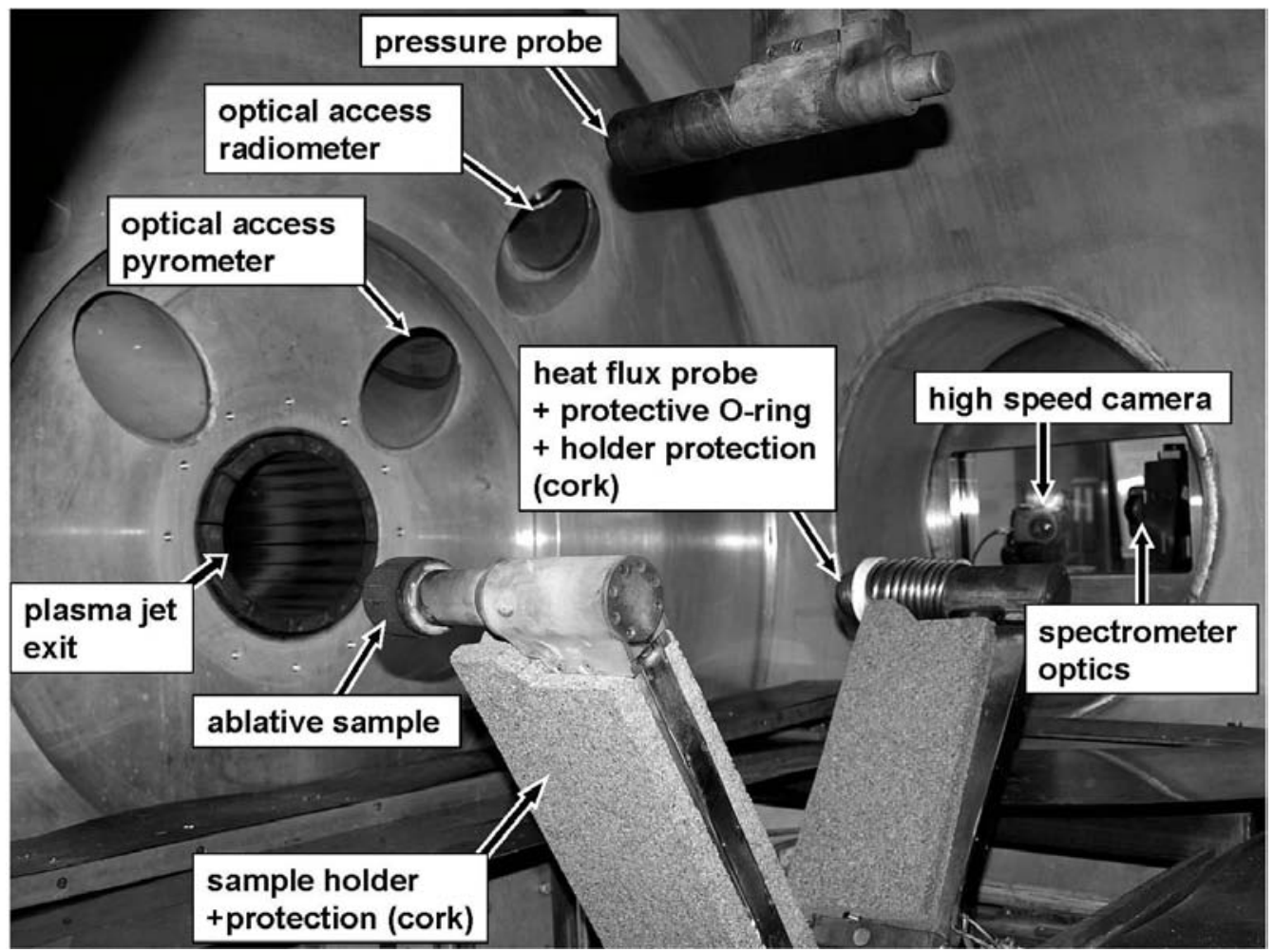

Fig. 6. Intrusive and non-intrusive measurement techniques applied

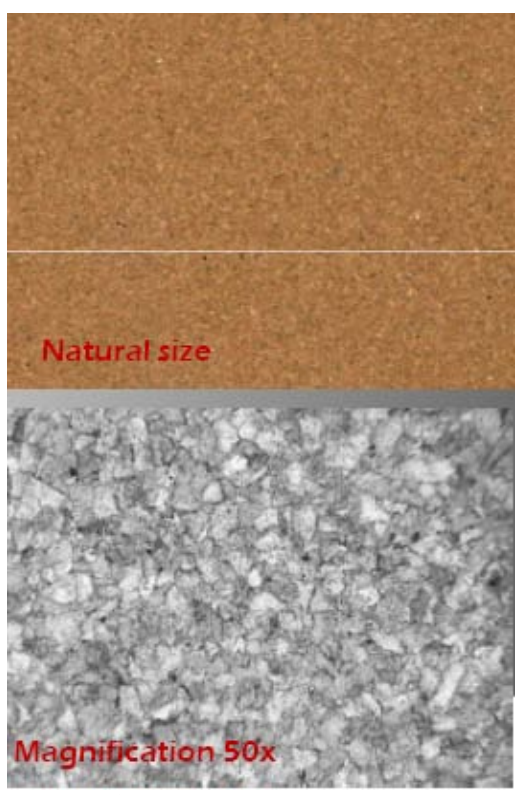

Fig. 7. Close-up and magnified views of Amorim P50
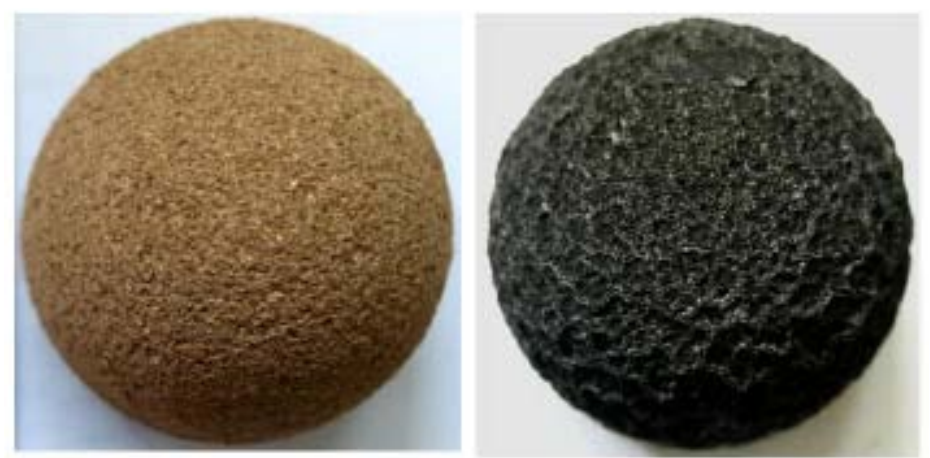

Fig. 8. Amorim P50 samples before (left) and after (right) test 


\section{Experimental Results}

Several Amorim P50 cork composite ablative thermal protection materials are tested at $1 \mathrm{MW} / \mathrm{m}^{2}$ and $3 \mathrm{MW} / \mathrm{m}^{2}$ heat flux conditions in the Plasmatron facility. The heat flux is measured by a water cooled (350K) copper calorimeter, which is highly catalytic with respect to oxygen and nitrogen recombinations. The mass flow rate of air is kept at $16 \mathrm{~g} / \mathrm{s}$ and the static pressure of the test chamber at 100 mbar. The Amorim P50 is a high strength cork composition made with 0.5 to 1 mm cork granule and a special binder for high heat resistance. It has a specific heat of $2018 \mathrm{~J} /(\mathrm{kg} \mathrm{K})$ within a temperature range of 38 to $316^{\circ} \mathrm{C}(100$ to $600 \mathrm{~F})$ and a thermal conductivity of $0.0820 \mathrm{~W} /(\mathrm{K} \mathrm{m})$ at $38^{\circ} \mathrm{C}(100 \mathrm{~F})$ and $0.0822 \mathrm{~W} / \mathrm{m} / \mathrm{K}$ at $260{ }^{\circ} \mathrm{C}(500 \mathrm{~F})$. Figure 7 shows a close-up view and the magnified view of the Amorim P50 sheet. The P50 samples tested in the Plasmatron facility are $50 \mathrm{~mm}$ diameter hemispherical samples, as shown in Fig. 8 (front view), before and after exposure to the air plasma flow.

Two type $\mathrm{K}$ thermocouples are utilized to measure the temperature inside the test sample $10 \mathrm{~mm}$ and $20 \mathrm{~mm}$ behind the stagnation point, as shown in Fig. 9. These measurements are very useful in determining the thermal efficiency of the ablative material, where the inner (back) temperature of the sample is required to be low despite the high front surface temperature. Figure 10 presents typical results from the two thermocouples utilized for the condition of $3 \mathrm{MW} / \mathrm{m}^{2}$ heat flux. Following the ignition of the Plasmatron facility, the sample starts heating, not because of ablation or exposure to plasma flow, but simply because it is in the close vicinity of a high temperature high enthalpy plasma flow. The Plasmatron facility is stopped after 30 seconds of exposure. The 30 second exposure to plasma is shown in Fig. 10 by the pink rectangular section, where no change in the slope of the temperature curve is observed, indicating that the observed temperature rise is due to heating from the side, not from the stagnation point. The increase in the inner temperature value is more significant after the 30 second exposure to plasma flow. This shows that the heat experienced by the stagnation point of the sample is transferred to the inner regions by a significant time delay, which is a very promising property of the tested thermal protection material.

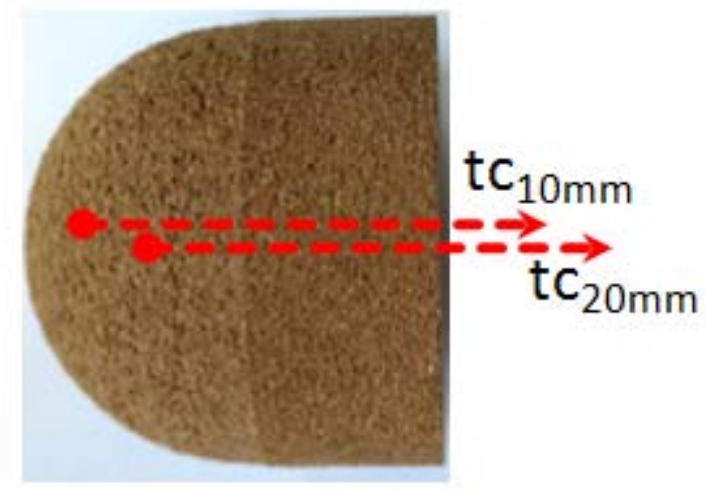

Fig. 9. Location of the two thermocouples measuring the temperature $10 \mathrm{~mm}$ and $20 \mathrm{~mm}$ inside the sample 


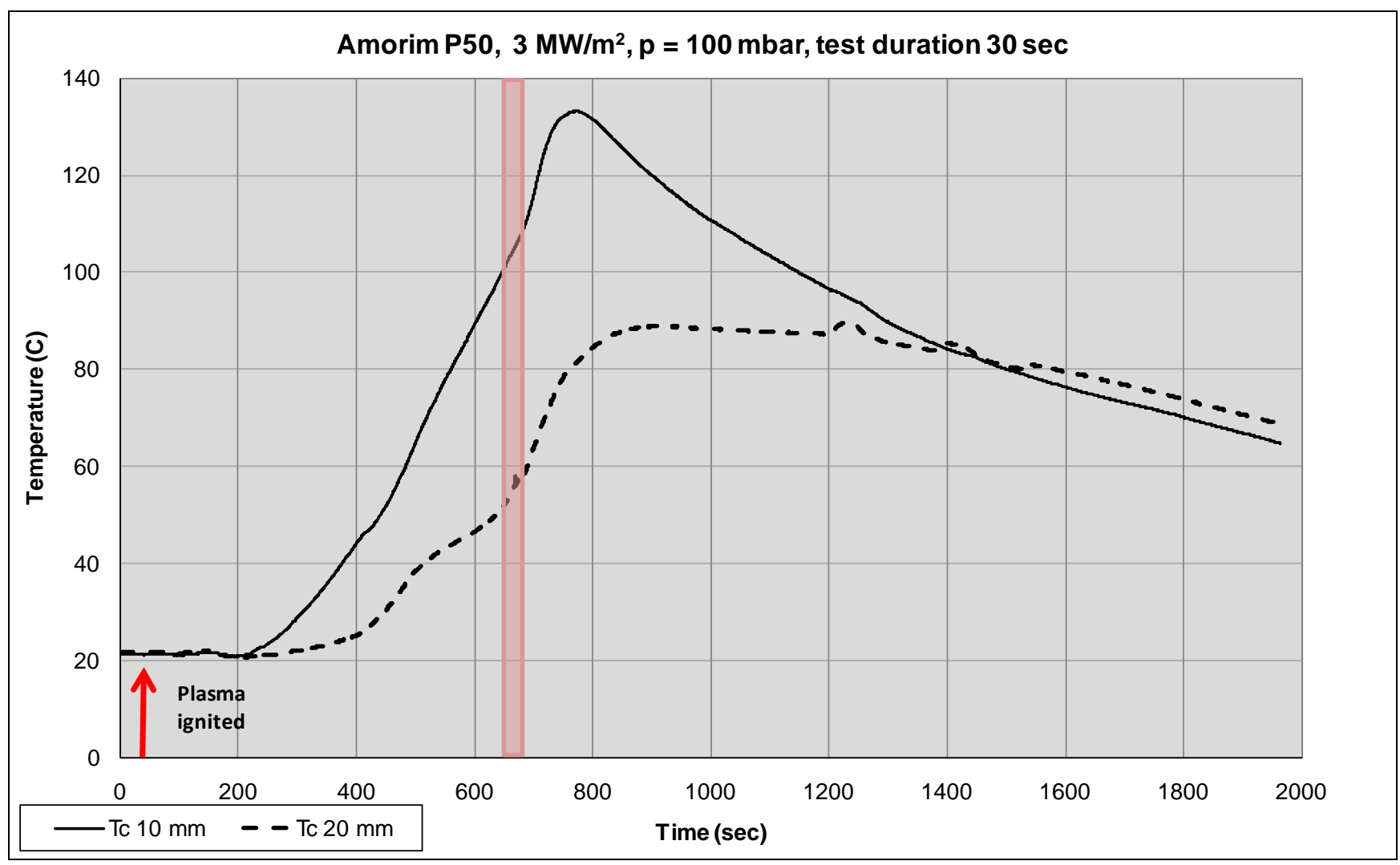

Fig 10. Temperature change inside the sample before, during and after exposure to plasma flow

Although the thermocouples read between $100^{\circ} \mathrm{C}$ and $130^{\circ} \mathrm{C}$ maximum inside the sample during and after the exposure to plasma flow at $3 \mathrm{MW} / \mathrm{m}^{2}$ heat flux, the front surface temperature recorded by the two-colour pyrometer and the other infrared thermography devices are much higher. Figures 11 and 12 present the front face temperature values as a function of time during the 30 seconds exposure to plasma flow, as observed by the two colour pyrometer, the infrared camera and the radiometer, for $1 \mathrm{MW} / \mathrm{m}^{2}$ and $3 \mathrm{MW} / \mathrm{m}^{2}$ incident heat flux conditions, respectively. The average front surface temperature is $1650{ }^{\circ} \mathrm{C}$ for the $1 \mathrm{MW} / \mathrm{m}^{2}$ heat flux condition and it is $2300{ }^{\circ} \mathrm{C}$ for the $3 \mathrm{MW} / \mathrm{m}^{2}$ heat flux condition, as recorded by the calibrated two-colour pyrometer.

The front face temperatures measured by the two-colour pyrometer, the infrared camera and the radiometer are plotted as a function of time in Figs. 11 and 12. For both plots, $t=0$ corresponds to the injection of the ablative sample into the plasma flow and the test duration is 30 seconds. For both cases, the pyrometer values do not need any correction as the pyrometer is calibrated for the test conditions and it is theoretically independent of the emissivity of the test sample. However, the infrared camera and the radiometer readings need to be corrected taking the emissivity into account. The infrared values presented in Fig. 11 (for $1 \mathrm{MW} / \mathrm{m}^{2}$ heat flux condition) are corrected assuming a factor of 0.66 for the transmissivity of the ZnSe $(\tau)$ window multiplied by the emissivity $(\varepsilon)$ of the test sample (where the radiation intensity is $I=\sigma . T . \varepsilon . T^{4}$ ). On the other hand, a factor of 0.64 is employed to correct the infrared camera results for the case of $3 \mathrm{MW} / \mathrm{m}^{2}$ presented in Fig. 12 . The transmissivity of the ZnSe window employed in front of the infrared camera is in the order of 0.95 for the wavelength range of $8-9 \mu \mathrm{m}$, as presented in Fig. 13. Considering a transmissivity of 0.95 for the ZnSe window, the emissivity measured by the infrared camera corresponds to 0.70 for the $1 \mathrm{MW} / \mathrm{m} 2$ heat flux case (1650 C temperature) and to $0.67 \mathrm{for}$ the $3 \mathrm{MW} / \mathrm{m} 2 \mathrm{heat}$ flux case (2300 C temperature). A similar analysis can be performed by comparing the results of the radiometer to the results of the pyrometer, employing the methodology proposed by [9]. In accordance with this methodology, the emissivity is calculated to be $0.73( \pm 0.08)$ for the $1 \mathrm{MW} / \mathrm{m} 2$ heat flux case $(1650 \mathrm{C}$ temperature), and to be $0.68( \pm 0.04)$ for the $3 \mathrm{MW} / \mathrm{m} 2$ heat flux case (2300 C temperature). These values are in agreement with those obtained from the comparison of the infrared camera results with the pyrometer results. In the plots of Fig. 11 and Fig. 12, both uncorrected and corrected radiometer results (by the mentioned emissivity values) are presented. It should be noted that the radiometer emissivity values do not need any correction for the transmissivity of the KRS-5 window, as this is already taken into consideration during the calibration of the radiometer. 


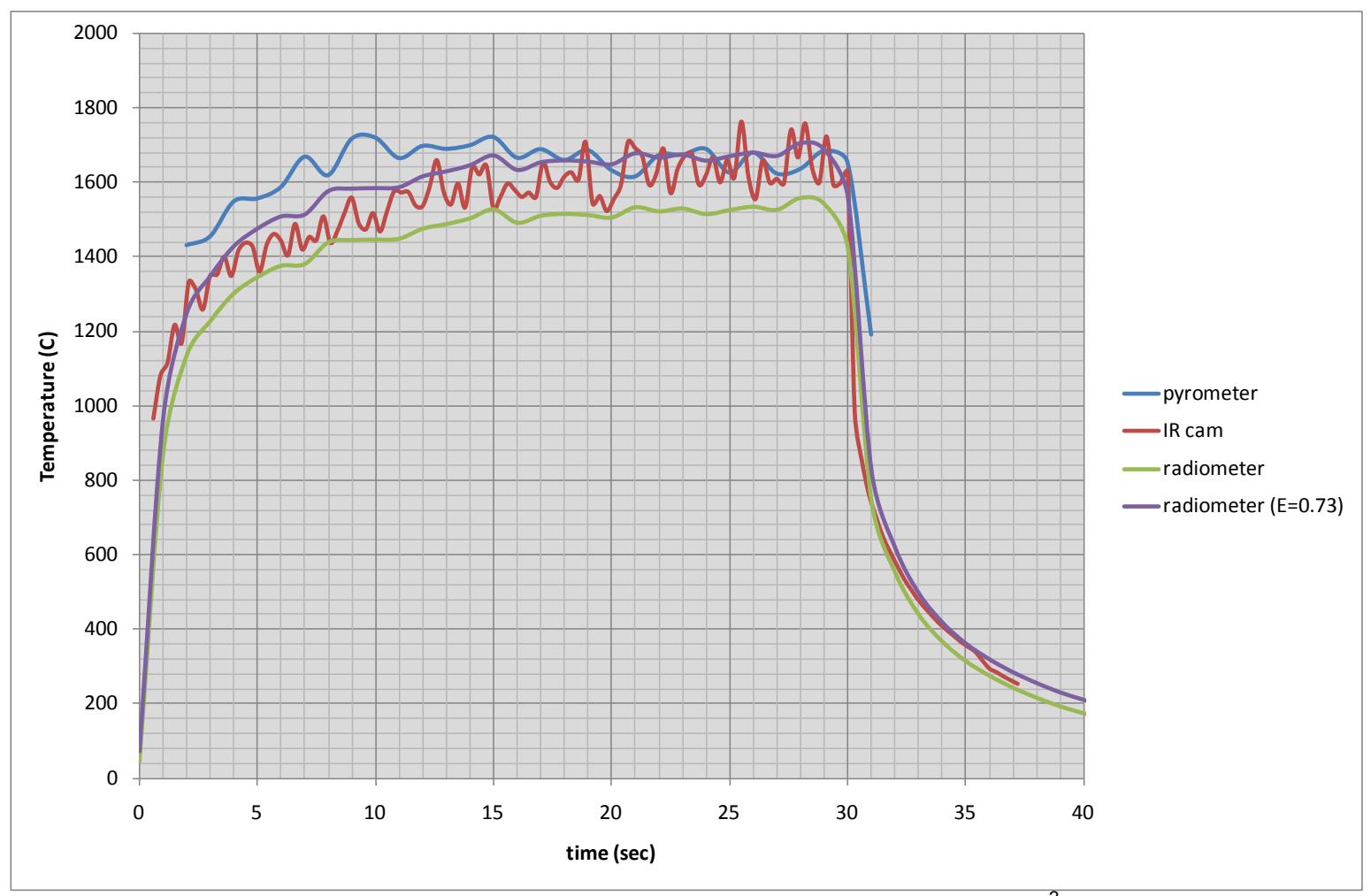

Fig 11. Front face temperatures for the test at $1 \mathrm{MW} / \mathrm{m}^{2}$ heat flux

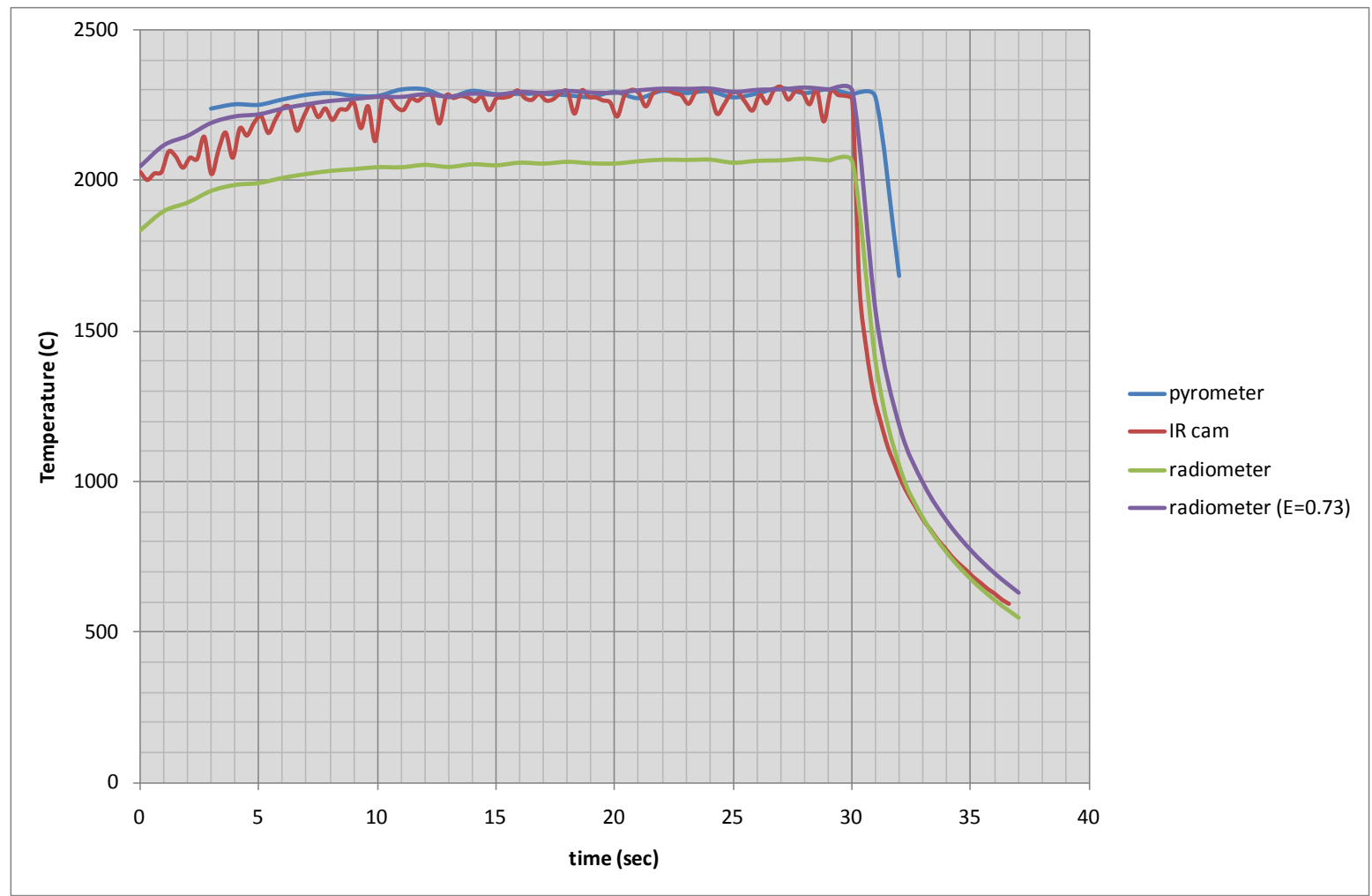

Fig 12. Front face temperatures for the test at $3 \mathrm{MW} / \mathrm{m}^{2}$ heat flux 


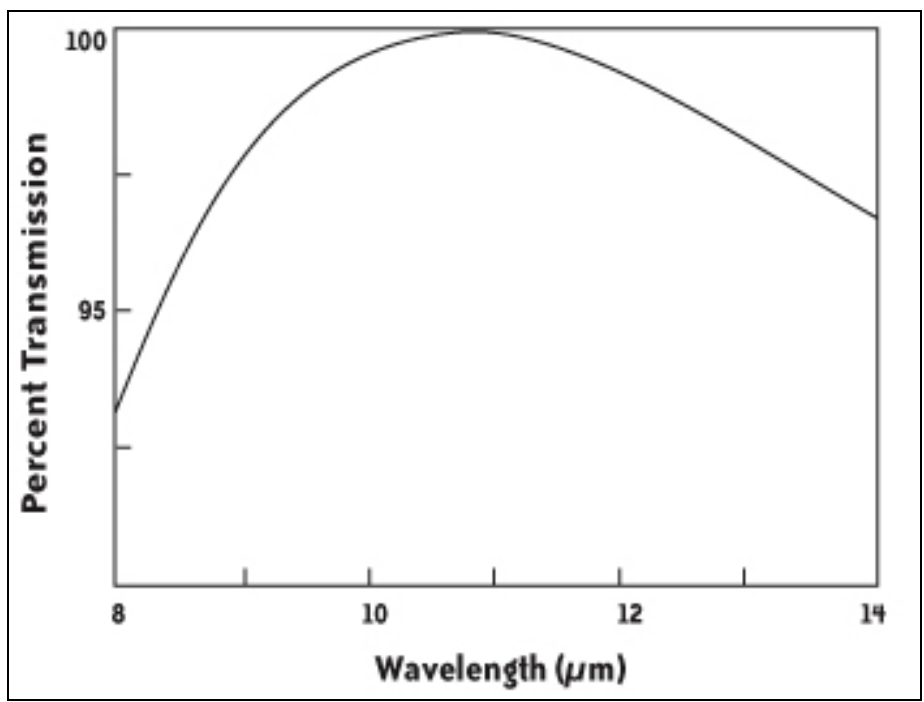

Fig 13. Transmissivity of the ZnSe window

\section{Conclusion}

The development of a multi-disciplinary experimental campaign is carried out at the Plasmatron facility of the von Karman Institute for the testing, analysis and qualification of ablative test samples. Among many measurement techniques utilized for this campaign, the measurement of the front surface temperature of the ablative test samples by infrared thermography is a technique of major importance. Amorim P50 cork composite samples are tested at $1 \mathrm{MW} / \mathrm{m}^{2}$ and $3 \mathrm{MW} / \mathrm{m}^{2}$ heat flux conditions with air plasma at 100 bar static pressure. Other than type $\mathrm{K}$ thermocouples measuring the inner temperature of the test samples, a two-colour pyrometer, an infrared camera and a radiometer are used for temperature determination. The pyrometer can detect the most reliable surface temperature values as it is independent of the emissivity of the test material. However the pyrometer measurements lack the information of the emissivity of the material at test conditions and they are only valid above a certain temperature (1300 C for this case). The emissivity values for the two test cases are determined comparing the infrared camera and pyrometer data, as well as by comparing the radiometer values and the pyrometer measurements. The emissivity values obtained from the two methods agree well if one considers the transmissivity of the ZnSe window. The advantage of utilising an infrared camera is that it supplies spatially resolved thermal information, although it has higher noise level as can be observed in Figures 11 and 12. On the other hand, although the radiometer supplies an averaged thermal response over a certain area (similar to the pyrometer), it provides low noise values.
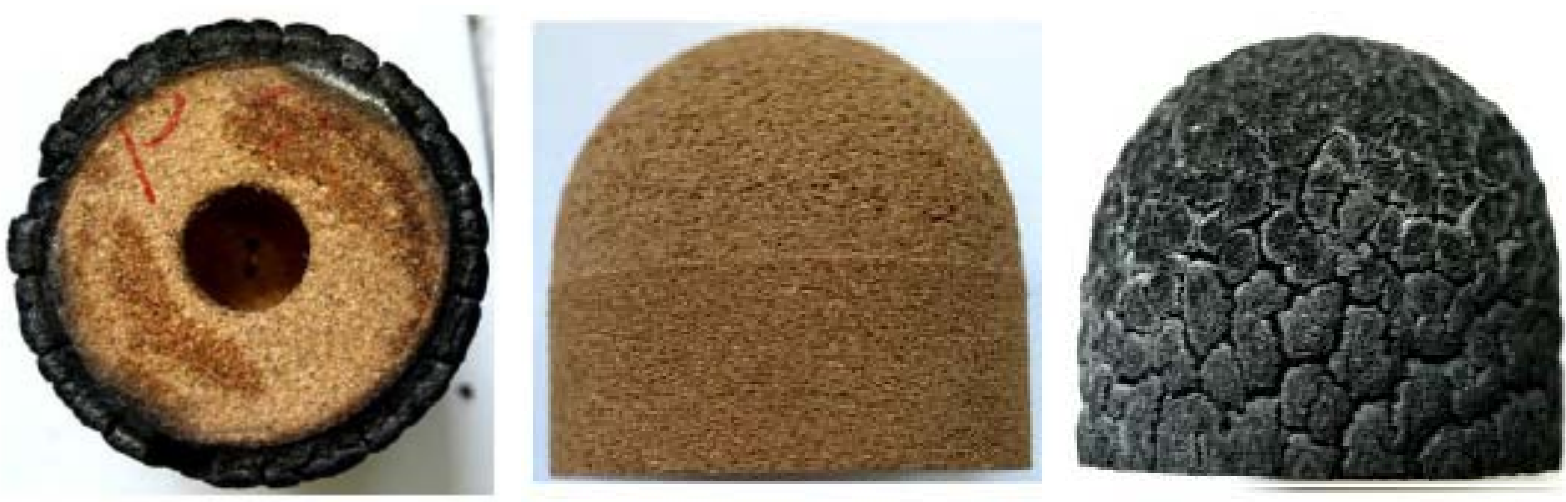

Fig 14. Back view (left) and side view (right) of test samples before (only side view) and after test 
Finally, it is interesting to observe the relatively very low temperature values inside the samples measured by thermocouples and compared to front face temperatures. This is believed to be due to conversion of the incident heat flux into ablation and sublimation of the ablative material. The ablation mechanism takes place in a very controlled manner and at a certain temperature, avoiding the heat wave to penetrate deeper into the material. An evidence for this assumption can be seen in Fig. 14, where the back view of a tested sample is shown. The black char layer (ablated section) penetrates into the material following a very uniform pattern.

\section{Acknowledgments}

The authors are thankful to Alexandre Pereira from Amorim Cork Composites for supplying the Amorim P50 composite cork samples. The effort published in this article is sponsored by the Air Force Office of Scientific Research, Air Force Material Command, USAF, under grant number FA8655-08-1-3069. The U.S. Government is authorized to reproduce and distribute reprints for Government purpose notwithstanding any copyright notation thereon.

\section{REFERENCES}

[1] Asma, C. O., Thoemel, J., Paris, S., Tirtey, S., Chazot, O. "Utilization of Infrared Thermography to Investigate Atmospheric Entry Aerothermodynamics of Space Vehicles at von Karman Institute," 9th International Conference on Quantitative InfraRed Thermography, 2-5 July 2008, Krakow, Poland.

[2] Howe, J., "Hypervelocity Atmospheric Flight: Real Gas Flow Fields", NASA TM 101055, 1989.

[3] Chen, Y. K., and Milos F. S., "Two-Dimensional Implicit Thermal Response and Ablation Program for Charring Materials", Journal of Spacecraft and Rockets, Vol.38, No. 4, July August 2001.

[4] Metzger, J. W., Engel, M. J., and Diaconis, N. S., "Oxidation and Sublimation of Graphite in Simulated Re-entry Environments", AIAA Journal, Vol. 5, No. 3, March 1967.

[5] Bottin, B., et al,"The VKI Plasmatron Characteristics and Performance", RTO AVT Course on "Measurement Techniques for High Enthalpy and Plasma Flows", Belgium, October 25-29, 1999.

[6] Erzincanli, B., "Ablative Material Testing in Plasmatron", VKI Project Report, PR 2009-24, von Karman Institute, Brussels, Belgium, July 2009.

[7] Vancrayenest B., and Fletcher D. G., "Investigation of the Thermochemistry of Ablation of Graphite for Planetary Entry Applications", 38th AIAA Thermophysics Conference Meeting and Exhibit, Ontorio, Canada June 6-9, 2005.

[8] Vancreyenest B. and Fletcher D.G., "Emission Spectroscopic Survey of Graphite Ablation in the VKI Plasmatron", 9th AIAA/ASME Joint Thermophysics and Heat Transfer Conference, San Francisco USA, June 5-8, 2006.

[9] Balat-Pichelin, M., Robert, J.F., Sans, J.L., "Emissivity measurements on carbon-carbon composites at high temperature under high vacuum", Applied Surface Science 253 (2006) 778-783, Oct 2005. 